\title{
HIGHLIGHTS
}

PEDIATRICS

\section{Optimizing caudal analgesia for minor urologic procedures}

The research efforts of two separate groups have generated novel findings that should markedly improve management of pediatric postoperative pain.

Control of pain in children who have undergone minor urologic operations is often reactive, involving oral administration of analgesics in response to patientreported distress. Support for a more preemptive approach comes from two new investigations-one of boys undergoing hypospadias repair, and another of children undergoing orchiopexy.

A pilot study of 17 boys whose hypospadias was repaired at the Radboud University Medical Centre in The Netherlands has shown a surprisingly long duration of caudal analgesia. Intraoperative administration of a levobupivacaine caudal block $(0.5 \mathrm{ml} / \mathrm{kg})$ was the only pain relief needed by $60 \%$ of patients until discharge on the morning following their operation. The higher of the two doses of levobupivacaine tested

Original articles Thies, K.-C. et al. Longer than expectedduration of caudal analgesia with two different doses of levobupivacaine in children undergoing hypospadias repair. J. Pediatr. Urol. 6, 585-588 (2010) | Hong, J.-Y. et al. Effect of dexamethasone in combination with caudal analgesia on postoperative pain control in day-case paediatric orchiopexy. Br. J. Anaesthes. 105, 506-510 (2010)
$(0.375 \%$ or $0.125 \%)$ was associated with an $85 \%$ pain-free rate.

Erim Erdem, commenting on the Faculty of 1000 website, lauds this preemptive approach. "The long-lasting effect of caudal analgesia is clinically very important, not only for hypospadias repair, but also for other penile operations such as circumcision," he asserts.

What of those patients for whom caudal block does not provide sufficient pain relief? The findings of a team based in Seoul, Republic of Korea, indicate that a single intravenous dose of the steroid dexamethasone can markedly reduce the need for rescue analgesia.

For their double-blind trial, the investigators randomized 77 boys aged 1-5 years undergoing orchiopexy as day patients to receive either $0.5 \mathrm{mg} / \mathrm{kg}$ dexamethasone or an equal volume of saline intraoperatively. Caudal analgesia $(1.5 \mathrm{ml} / \mathrm{kg}$ of ropivacaine $0.15 \%)$ was then administered prior to incision.

Just $8 \%$ of boys in the dexamethasone group required fentanyl in the postoperative care unit, compared with $39 \%$ of controls. Postdischarge use of acetaminophen was also markedly less frequent in the dexamethasone group (24\% versus $64 \%$ ). There was no difference in the incidence of adverse events between the study arms.

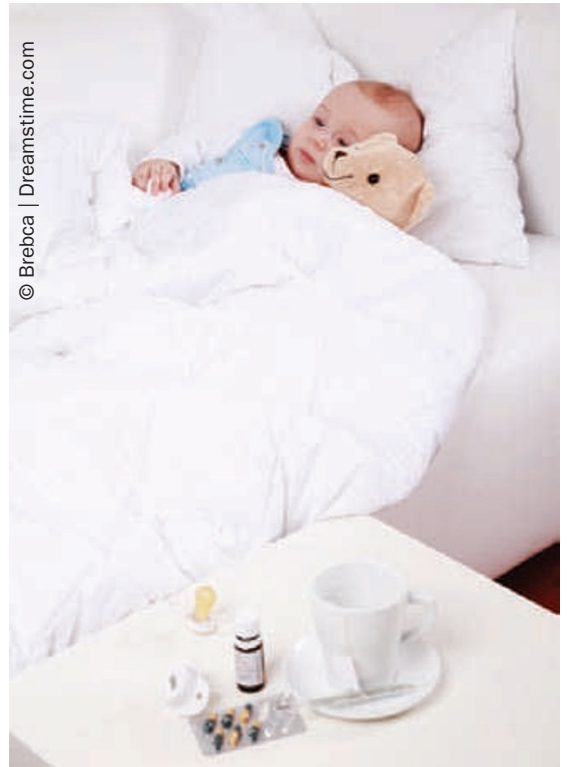

Faculty of 1000 evaluators Julia Siebert and Bernadette Veering note that use of dexamethasone to prolong the pain relief conferred by caudal analgesia has the advantage of antiemesis while sparing young patients the adverse effects of opioids. They hope that "a consensus regarding the routine use of [this steroid] in children" can soon be reached.

Suzanne J. Farley 